\title{
ERRATUM
}

\section{Non-Anatomic Proximal Realignment for Recurrent Patellar Dislocation Does Not Sufficiently Prevent Redislocation}

Due to an oversight of the manuscript, some minor errors were published in journal "The Open Orthopaedics Journal" 2012; 6: 114-117.

At the time of the operation Dr. Turgay Efe was affiliated to the department of Trauma, Hand and Reconstructive Surgery. Due to a thorough review of all operation reports it became clear that surgeries were performed by more than two surgeons. 